\title{
Desenvolvimento de um coletor solar plano para um sistema de secagem utilizando o planejamento experimental fatorial
}

\author{
Development of a flat solar collector for a drying system using factorial experimental design \\ Desarrollo de un colector solar plano para un sistema de secado mediante disenõ experimental \\ factorial
}

Recebido: 03/06/2021 | Revisado: 09/06/2021 | Aceito: 10/06/2021 | Publicado: 29/06/2021

\author{
Maria de Sousa Leite Filha \\ ORCID: https://orcid.org/0000-0003-4576-3979 \\ Universidade Federal de Campina Grande, Brasil \\ E-mail: mariadslfilha@gmail.com \\ Michel Barros Silva \\ ORCID: https://orcid.org/0000-0003-2303-4943 \\ Universidade Federal de Campina Grande, Brasil \\ E-mail: michelbarrosufcg@hotmail.com \\ Ítalo de Andrade Gomes \\ ORCID: https://orcid.org/0000-0001-6537-4394 \\ Universidade Estadual da Paraíba, Brasil \\ E-mail: eng.iagomes@gmail.com \\ Marcelo Bezerra Grilo \\ ORCID: https://orcid.org/0000-0002-6415-5058 \\ Universidade Federal de Campina Grande, Brasil \\ E-mail: griloufcg@yahoo.com.br \\ Nancy Lima Costa \\ ORCID: https://orcid.org/0000-0001-5313-5070 \\ Universidade de Pernambuco, Brasil \\ E-mail: Nancy.costa@upe.br \\ João André Soares e Sousa da Conceição \\ ORCID: https://orcid.org/0000-0002-4091-2587 \\ Universidade Federal de Campina Grande, Brasil \\ E-mail: joao.andre@estudante.ufcg.edu.br \\ Vitor Leão Santana \\ ORCID: https://orcid.org/0000-0002-7663-9471 \\ Universidade Federal de Campina Grande, Brasil \\ E-mail: vitorlsantana@gmail.com \\ Jonas Fernando de Souza Fernandes \\ ORCID: https://orcid.org/0000-0002-0444-0391 \\ Universidade Federal de Campina Grande, Brasil \\ E-mail: jonasengmecanica@gmail.com
}

\begin{abstract}
Resumo
O presente trabalho tem o objetivo de determinar a configuração ótima para um coletor solar plano de baixo custo, que fosse de fácil construção e manutenção e pudesse ser utilizado na secagem de frutas pela agricultura familiar. Para tanto aplicou-se a metodologia do planejamento experimental fatorial devido sua confiabilidade e por avaliar ao mesmo tempo o efeito de diversos fatores ou variáveis, a partir de um número mínimo de experimentos. Foram determinadas as variáveis a serem estudadas, seguindo com a realização do conjunto de experimentos necessários e com a análise das variáveis estatisticamente significativas para determinação da configuração ótima do coletor solar. Os resultados experimentais determinaram como sendo a configuração otimizada: Cobertura transparente de policarbonato alveolar e utilização de uma telha de zinco pintada de preto fosco como capacitor térmico. $\mathrm{O}$ cálculo do rendimento térmico do equipamento foi usando sua definição como sendo a razão entre a potência entregue pelo sistema e a potência disponível ao mesmo e o resultado obtido para a configuração ótima foi de $42,00 \%$. O planejamento experimental fatorial desenvolvido possui acurácia de $96,10 \%$ e se mostrou significativo e preditivo.

Palavras-chave: Coletor solar; Planejamento experimental fatorial; Rendimento térmico; Energia solar.
\end{abstract}

\footnotetext{
Abstract

This work aims to determine the optimal configuration for a low-cost flat solar collector, which is easy to build and maintain and can be used for drying fruit by family farmers. For this purpose, the factorial experimental design methodology was applied due to its reliability and for evaluating at the same time the effect of several factors or
} 
variables, from a minimum number of experiments. The variables to be studied were determined, following with the carrying out of the necessary set of experiments and with the analysis of statistically significant variables to determine the optimal configuration of the solar collector. The experimental results determined as the optimized configuration: Transparent alveolar polycarbonate roofing and use of a matte black painted zinc tile as a thermal capacitor. The calculation of the equipment's thermal efficiency was using its definition as the ratio between the power delivered by the system and the power available to it, and the result obtained for the optimal configuration was $42.00 \%$. The factorial experimental design developed has an accuracy of $96.10 \%$ and proved to be significant and predictive.

Keywords: Solar collector; Factorial experimental planning; Thermal yield; Solar energy.

\section{Resumen}

El presente trabajo tiene como objetivo determinar la configuración óptima para un colector solar plano de bajo costo, que sea fácil de construir y mantener y pueda ser utilizado para secar fruta por agricultores familiares. Para ello, se aplicó la metodología de diseño experimental factorial por su confiabilidad y para evaluar al mismo tiempo el efecto de varios factores o variables, a partir de un número mínimo de experimentos. Se determinaron las variables a estudiar, siguiendo con la realización del conjunto de experimentos necesarios y con el análisis de variables estadísticamente significativas para determinar la configuración óptima del colector solar. Los resultados experimentales se determinaron como configuración optimizada: Cubierta de policarbonato alveolar transparente y uso de una loseta de zinc pintada de negro mate como condensador térmico. El cálculo de la eficiencia térmica del equipo se definió como la relación entre la potencia entregada por el sistema y la potencia disponible para el mismo, y el resultado obtenido para la configuración óptima fue 42,00\%. El diseño experimental factorial desarrollado tiene una precisión del 96,10\% y resultó significativo y predictivo.

Palabras clave: Colector solar; Disenõ experimental factorial; Eficiencia térmica; Energía solar.

\section{Introdução}

O desenvolvimento de secadores solares cada vez mais eficientes e acessíveis aos pequenos agricultores vem sendo objeto de estudo de várias instituições internacionais e brasileiras. No Nordeste brasileiro destacam-se os trabalhos desenvolvidos pelo Grupo de Pesquisa em Energia e Desenvolvimento Sustentável (GEDS) da UFCG, que há mais de 10 anos vem desenvolvendo e testando sistemas de secagem solar construído com materiais de baixo custo, sustentáveis e de fácil manutenção. Na Universidade Federal do Rio Grande do Norte (UFRN) e na Universidade Federal de Sergipe (UFS) ocorre o desenvolvimento e aprimoramento de diversos secadores solares usando materiais sustentáveis, obtendo-se resultados promissores.

Segundo Oliveira et al., (2018) o coletor solar é o principal componente de um secador solar, uma vez que atua diretamente na captação da radiação solar e sua posterior conversão em energia térmica. Essa radiação ao incidir sobre o coletor de energia solar atravessa a cobertura transparente sendo absorvida no interior do coletor solar, tendo como consequência o aumento da temperatura no interior do mesmo, fenômeno este conhecido como efeito estufa.

Segundo Silva e Sousa (2020), a energia solar se tornou uma das formas de energia alternativa mais utilizadas, por ser uma fonte renovável e não poluente.

O Brasil possui áreas com valores de radiação solar comparáveis às melhores do mundo, com destaque para a região Nordeste. (Cresesb, 2019).

Atualmente a pesquisa na área científica tem proporcionado significativos avanços tecnológicos e, com isso, tem gerado um número desmedido de dados e informações. Segundo Pereira-Filho et al., (2002), para a devida análise destes dados é indispensável a utilização de ferramentas estatísticas. Segundo Rodrigues e Iemma (2014) isto tem levado profissionais de diferentes formações a buscarem técnicas sistemáticas de planejamento de experimentos que, para Montgomery (2017) são utilizadas para melhorar as características qualitativas dos produtos ou processos.

Planejar experimentos, de certo modo, consiste em estabelecer uma sequência organizada de obtenção de dados experimentais visando atingir objetivos pré-determinados. De acordo com Brasil et al. (2007), as desvantagens dos procedimentos de análise univariado, como o a falta de análise de interações entre as variáveis, pode resultar numa otimização inadequada que poderia ser obtido empregando-se sistemas de análise multivariados. 
Assim sendo, diante da relevância do coletor solar para o bom funcionamento de um secador solar, da disponibilidade de radiação solar no Nordeste brasileiro e da importância de se aplicar um método de coleta e análise de dados eficiente e confiável este trabalho tem o objetivo de aplicar a metodologia do planejamento experimental fatorial para a otimização de um coletor solar plano de baixo custo, que fosse de fácil construção e manutenção e pudesse utilizado na secagem de frutas pela agricultura familiar.

Diversos modelos de coletores solares já foram desenvolvidos e a variedade de materiais também é abrangente.

Jacob (2016) adaptou e utilizou uma antena parabólica de 2,20 m de diâmetro como aquecedor solar de água de fluxo contínuo, obtendo rendimento térmico máximo de 44,45\% num dia sem nuvens e de intensidade de radiação solar de $800 \mathrm{~W} / \mathrm{m}^{2}$.

Nunes (2016) desenvolveu um secador solar de frutas constituído por um coletor solar com volume interno de 176 litros e área de incidência 1,0 $\mathrm{m}^{2}$ feito de MDF Ultra e com cobertura transparente de policarbonato, obtendo rendimento térmico médio de $19,60 \%$.

Coelho et al., (2019) desenvolveram um secador solar de exposição direta, ou seja, aquele em que coletor solar e câmara de secagem são o mesmo equipamento, a partir de um tambor metálico de 200 litros que foi cortado ao meio e deu origem a dois secadores.

Fernandes (2021) desenvolveu dois secadores solares de exposição direta com área de exposição de $0,287 \mathrm{~m}^{2}$ e o volume interno de $0,055 \mathrm{~m}^{3}$ (55 litros). Os equipamentos foram construídos de MDF de $15 \mathrm{~mm}$ de espessura obtendo rendimento térmico médio de $36,3 \%$ e de $32,6 \%$.

Gomes (2021) aplicou a metodologia do planejamento experimental fatorial na determinação da configuração ótima de um secador solar de exposição indireta e a melhor configuração obtida para o coletor solar foi: material isolante a madeira de Pinus, cobertura de policarbonato alveolar e volume interno de 100 litros. O rendimento termodinâmico médio para esta configuração de coletor foi $20,16 \%$.

Segundo Almeida et al. (2018), a maioria das propriedades rurais do Brasil é constituída por produtores que trabalham em pequena escala, tendo como mão de obra predominante o núcleo familiar, denominando a agricultura familiar.

O uso de sistemas de secagem solar de produtos agrícolas é viável, econômico e ideal para agricultores em muitos países em desenvolvimento (Mustayen et al., 2014).

O coletor solar desenvolvido neste trabalho poderá ser integrado a uma câmara de secagem para compor um secador solar de frutas. Visando desenvolver um equipamento que pudesse ser aplicado na agricultura familiar é importante que sua construção seja simples e os materiais utilizados sejam acessíveis e de baixo custo.

\section{Metodologia}

Para determinar a melhor configuração do coletor solar foi utilizado o planejamento experimental fatorial por ser capaz de identificar os fatores ou combinação de fatores mais relevantes para a resposta do experimento (Peralta-Zamora et al. 2005; Fulano et al. 2008).

As principais características construtivas do coletor solar desenvolvido são:

- Dimensões internas: $0,80 \mathrm{~m}(\mathrm{~L})$ x $1,25 \mathrm{~m}$ (C) x 0,10 m (A). Totalizando um volume interno de $100 \mathrm{~L}$. A cobertura transparente possui área de $1 \mathrm{~m}^{2}$.

- Entrada e saída de ar medindo: 0,70 m x 0,05 m. Totalizando uma área de 0,035 $\mathrm{m}^{2}$.

- Corpo construído de polietileno estendido (isopor) de $0,025 \mathrm{~m}$ de espessura que funciona como isolante térmico, o torna mais leve e barato; 
- Revestido interno e externo de alumínio que funciona como trocador de calor além de auxiliar na sustentação da estrutura.

- Pintura internamente de preto fosco de modo intensificar a absorção da radiação solar e transformá-la em energia térmica.

A partir disso, propôs-se a metodologia do planejamento experimental fatorial para refinar outras especificações como material para a cobertura transparente e a inserção ou não de um capacitor térmico. As variáveis estudadas foram: capacitor térmico e cobertura transparente. A escolha destas variáveis deu-se pela possibilidade destas promoverem maior aumento de temperatura no coletor solar.

Testou-se como capacitor térmico uma telha ondulada de zinco pintada de preto fosco. A telha ondulada, mesmo possuindo as mesmas dimensões da base do coletor, possui maior área superficial e suas ondulações provocam maior turbulência do ar dentro do coletor, o que pode levar a maiores ganhos de temperatura.

Como cobertura transparente, foi utilizado o plástico estufa e policarbonato alveolar de $4 \mathrm{~mm}$. Nas coberturas foram utilizadas cantoneiras em $\mathrm{U}$ de $0,01 \mathrm{~m}$ de espessura para a moldura e cola de silicone para vedar a saída de ar. As coberturas possuem $1,30 \mathrm{~m}$ de comprimento e $0,85 \mathrm{~m}$ de largura.

A codificação das variáveis com seus respectivos níveis, no coletor solar, foram:

- Capacitor térmico (A):

- Cobertura transparente (B):
-1 [sem] e +1 [com];

-1 [plástico] e +1 [policarbonato];

Segundo Neves et al. (2002), o planejamento experimental fatorial completo é do tipo $\mathrm{b}^{\mathrm{k}}$, onde k representa o número de fatores (variáveis) e "b" o número de níveis fixados.

Como foram estudadas duas variáveis, o número de experimentos foi de $2^{2}=4$ e cada experimento contou com duas réplicas, totalizando 12 experimentos.

Na Figura 1 são apresentados os dois coletores solares construídos.

Figura 1: Coletor solar com cobertura de plástico estufa e policarbonato.
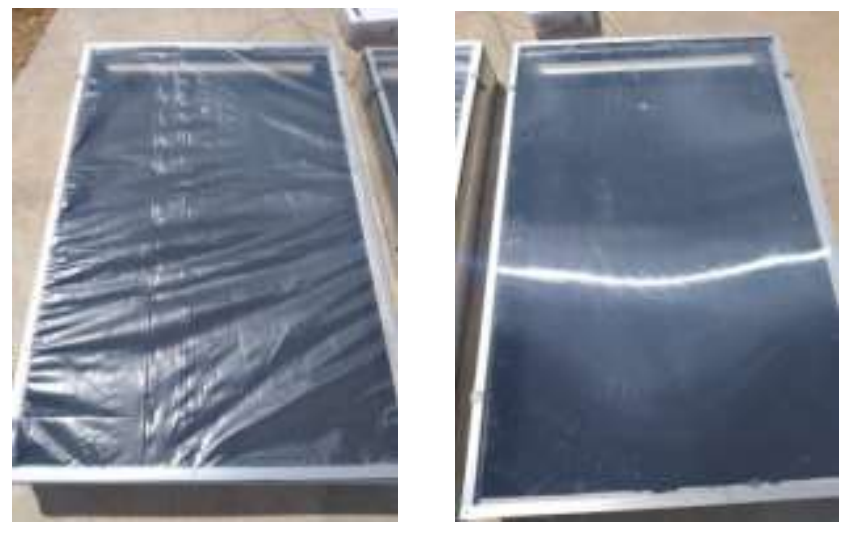

Fonte: Autores.

Nesta figura pode-se observar que dois coletores idênticos, em termos de construção, a parte interna pintada de preto fosco e as coberturas de plástico estufa e de policarbonato alveolar. 
Para o monitoramento das propriedades termodinâmicas do ar foi desenvolvido um sistema de medição e aquisição de dados (SMAD) por meio da utilização de uma plataforma de prototipagem eletrônica do tipo Arduino com sensores de medição de temperatura e umidade relativa do ar do tipo DHT22.

Entre os dias 01 e 30 de novembro de 2020 foram realizados testes experimentais na cidade de Pombal/PB. Cada coletor ficou exposto à radiação solar por 04 (quatro) horas, sempre das 10:00 às 14:00 que são os horários de maior incidência de radiação solar e consequente ganho de temperatura.

Segundo Grilo (2007), em cidades como Pombal/PB, os coletores de energia solar devem ficar voltados para o norte com um ângulo de aproximadamente $17^{\circ}$ a $22^{\circ}$ que corresponde a soma da latitude local ( $7^{\circ}$ sul, aproximadamente) mais um ângulo de $10^{\circ}$ ou $15^{\circ}$, com isto o coletor ficaria mais perpendicular a incidência de radiação solar pois compensaria a inclinação da Terra, visto que esta é de $23,5^{\circ}$.

Os dados de temperatura e umidade relativa do ar foram obtidos a cada minuto em seguida divididos em intervalos de uma hora (60 minutos), foram calculados a média, o desvio padrão e o coeficiente de variação dos dados. Estas informações são importantes para garantir que os experimentos sejam realizados sob mesmas condições e não acarretar prejuízo às análises. Na Tabela 1 constam os dados obtidos.

Segundo Freund (2006), o coeficiente de variação ou de dispersão expressa o desvio-padrão como uma porcentagem do que está sendo medido além de indicar a dispersão dos dados em torno da média.

Tabela 1: Média, desvio padrão e coeficiente de variação dos dados de temperatura e umidade relativa.

\begin{tabular}{c|c|c|c|c|c|c}
\hline & \multicolumn{3}{|c|}{ Temperatura } & \multicolumn{3}{c}{ Umidade relativa } \\
\hline Hora & Média & $\begin{array}{c}\text { Desvio } \\
\text { padrão }\end{array}$ & $\begin{array}{c}\text { Coeficiente } \\
\text { de variação }\end{array}$ & Média & $\begin{array}{c}\text { Desvio } \\
\text { padrão }\end{array}$ & $\begin{array}{c}\text { Coeficiente de } \\
\text { variação }\end{array}$ \\
\hline $10: 00-11: 00$ & 39,53 & 1,72 & $4 \%$ & 38,90 & 2,23 & $5,7 \%$ \\
\hline $11: 00-12: 00$ & 41,72 & 1,96 & $4,7 \%$ & 35,14 & 2,41 & $6,8 \%$ \\
\hline $12: 00-13: 00$ & 43,58 & 1,92 & $4,4 \%$ & 38,63 & 2,22 & $5,7 \%$ \\
\hline $13: 00-14: 00$ & 43,48 & 1,72 & $3,9 \%$ & 35,13 & 2,51 & $7,1 \%$ \\
\hline
\end{tabular}

Fonte: Autores.

A partir dos dados apresentados na Tabela 1 observa-se que o coeficiente de variação nos quatro intervalos de tempo de uma hora para os dados de temperatura e umidade relativa é de, no máximo 7,1\%. Assim, pode-se inferir que os experimentos foram realizados sob condições climáticas similares e que a interpretação dos resultados não fica prejudicada com o fato destes testes não terem sido realizados simultaneamente.

\subsection{Rendimento térmico do coletor solar}

A partir do conceito básico de rendimento termodinâmico, como sendo a razão entre a potência entregue pelo sistema e a potência disponível ao mesmo, Varella e Guerra (2014) afirmaram que o rendimento de um secador solar pode ser determinado pela Equação 1 


$$
\eta_{t}=\frac{P_{u}}{A-I} \cdot 100
$$

Onde,

$\eta_{\mathrm{t}}=$ rendimento do secador solar $[\%]$;

$P_{u}=$ potência transferida ao fluido de trabalho $[\mathrm{W}]$;

$A=$ área de cobertura transparente $\left[\mathrm{m}^{2}\right]$;

$I=$ radiação solar incidente $\left[\mathrm{W} / \mathrm{m}^{2}\right]$.

A potência transferida ao fluido de trabalho, também chamada de potência útil, é determinada pela Equação 2, e representa a quantidade de energia transferida ao fluido de trabalho, neste caso, o ar ambiente:

$$
P_{u}=\dot{m} \cdot c_{p} \cdot \Delta T
$$

Onde,

$\dot{m}=$ vazão mássica do fluido de trabalho $[\mathrm{kg} / \mathrm{s}]$;

$c_{\mathrm{p}}=$ calor específico do fluido de trabalho $[\mathrm{J} / \mathrm{kg} . \mathrm{K}]$;

$\Delta T=$ variação de temperatura $[\mathrm{K}]$.

Dessa forma, o rendimento térmico de um secador solar é expresso em função da potência útil, da radiação solar incidente, da área de cobertura transparente e, consequentemente, da vazão do fluido de trabalho, do calor específico do mesmo e da variação de temperatura obtida.

Os dados de radiação solar incidente utilizados são os disponibilizados pelo Instituto Nacional de Meteorologia $(\text { INMET })^{1}$ para radiação solar através do seu portal na internet.

A vazão mássica é dada pela Equação 3.

$$
\ddot{m}=v * A * \rho
$$

Onde,

$\nu=$ velocidade do ar $[\mathrm{m} / \mathrm{s}]$

$A$ = área da secção transversal da chaminé $\left[\mathrm{m}^{2}\right]$;

$\rho=$ massa específica do ar $\left[\mathrm{kg} / \mathrm{m}^{3}\right]$.

A variação de temperatura nos pontos de interesse, dado pela Equação 4, foram medidas em intervalos de 1 minuto, em seguida calculado sua média com um sistema de aquisição de dados.

\footnotetext{
${ }^{\square}$ Instituto Nacional de Meteorologia - INMET, órgão do Ministério da Agricultura, Pecuária e Abastecimento, que fornece informações meteorológicas à sociedade brasileira através das suas estações meteorológicas distribuídas pelo país, cujos dados estão disponíveis em <http://www.inmet.gov.br/portal/index.php?r=estacoes/estacoesAutomaticas>
} 


$$
\Delta T=T_{s}-T_{e}
$$

Onde,

$T_{s}=$ Temperatura de saída do coletor solar $[\mathrm{K}]$

$T_{e}=$ Temperatura de entrada do coletor solar $[\mathrm{K}]$.

\section{Resultados e Discussão}

Os resultados ora apresentados representam a aplicação da metodologia do planejamento experimental fatorial na determinação da configuração ótima de um equipamento capaz de elevar a temperatura do ar ambiente a níveis suficientes para realizar o processo de secagem solar.

A resposta estudada foi a diferença entre a temperatura ambiente (temperatura de entrada do coletor solar) e a temperatura de saída do coletor solar $(\Delta \mathrm{T})$.

Os 12 experimentos necessários para realizar um planejamento experimental completo $2^{2}$ com duas réplicas foram realizados entre os dias 01 e 30 de novembro de 2020 com condições climáticas de umidade relativa média ambiente de $34,54 \%$ e temperatura ambiente média de $42,08^{\circ} \mathrm{C}$. A matriz codificada do planejamento está na Tabela 2 com a respectiva resposta obtida em cada ensaio.

Tabela 2: Matriz do planejamento experimental fatorial.

\begin{tabular}{c|c|c|c|c|c}
\hline Experimento & $\mathbf{A}$ & $\mathbf{B}$ & $\begin{array}{c}\text { Resposta Experimento } \\
(\Delta \mathbf{T})\end{array}$ & $\begin{array}{c}\text { Resposta Réplica 1 } \\
\left(\Delta \mathbf{T}_{\mathbf{R} 1}\right)\end{array}$ & $\begin{array}{c}\text { Resposta Réplica 2 } \\
\left(\Delta \mathbf{T}_{\mathbf{R} 2}\right)\end{array}$ \\
\hline 1 & -1 & -1 & 27,83 & 24,87 & 26,81 \\
\hline 2 & +1 & -1 & 37,20 & 35,35 & 30,54 \\
\hline 3 & -1 & +1 & 22,01 & 23,00 & 24,48 \\
\hline 4 & +1 & +1 & 46,65 & 47,96 & 44,06 \\
\hline
\end{tabular}

Fonte: Autores.

Pode-se observar a partir dos dados da Tabela 2 a diferença de temperatura entre o ar ambiente e o ar de saída do coletor solar é de no mínimo $22,01^{\circ} \mathrm{C}$ e de no máximo $47,96^{\circ} \mathrm{C}$, sendo que nos experimentos 2 e 4 e suas réplicas são obtidos os maiores valores de ganho de temperatura.

Os resultados obtidos nos experimentos foram tratados pelo Design of Experimental (DOE) - planejamento experimental no software Statistic versão 10.0, permitindo, assim, extrair as informações estatísticas necessárias à interpretação e otimização do coletor solar para o sistema de secagem proposto.

Os testes realizados obtiveram um coeficiente de correlação ou porcentagem de variação explicada, que indica o quão próximos os dados experimentais estão dos previstos, de 96,10\%, o que é um bom resultado já que para este tipo de análise o coeficiente de correlação acima de 95\% é considerado satisfatório. O Gráfico 1 mostra os valores observados e previstos. 
Gráfico 1: Valores observados experimentalmente versus valores previstos.

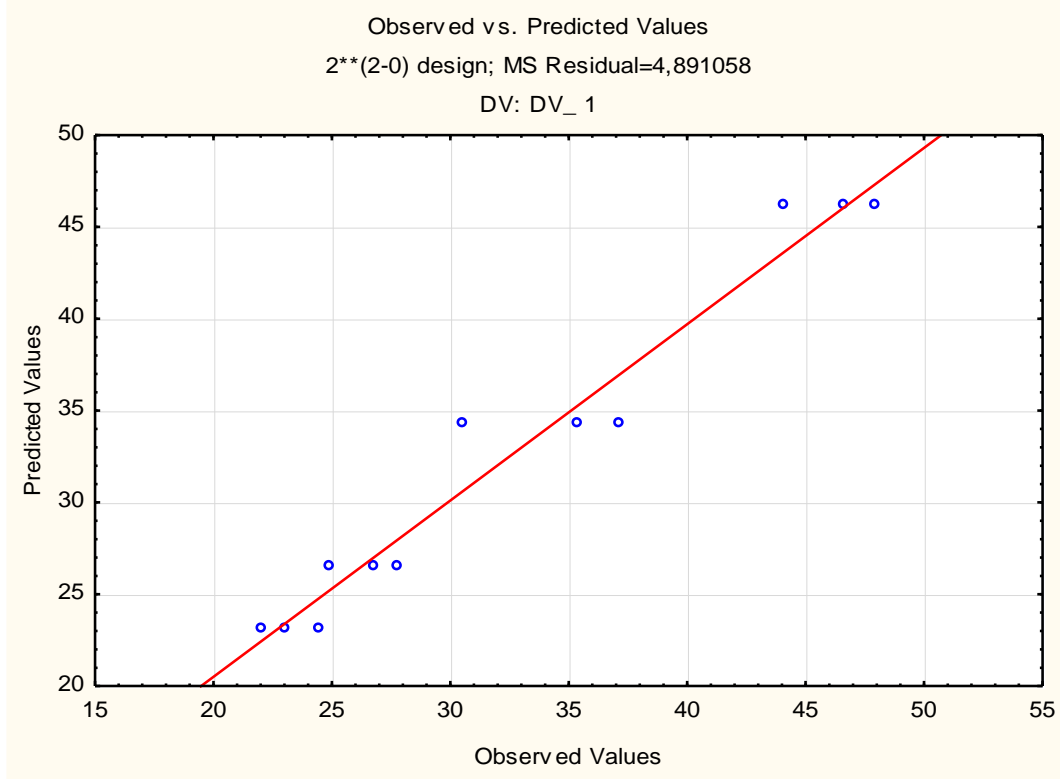

Fonte: Autores.

Nota-se, a partir do Gráfico 1 que os valores observados experimentalmente são bem ajustados à curva teórica de valores esperados.

A interpretação das variáveis com significância estatística para o processo pode ser feita observando o Gráfico de Pareto do experimento realizado (Gráfico 2).

Gráfico 2: Gráfico de Pareto.

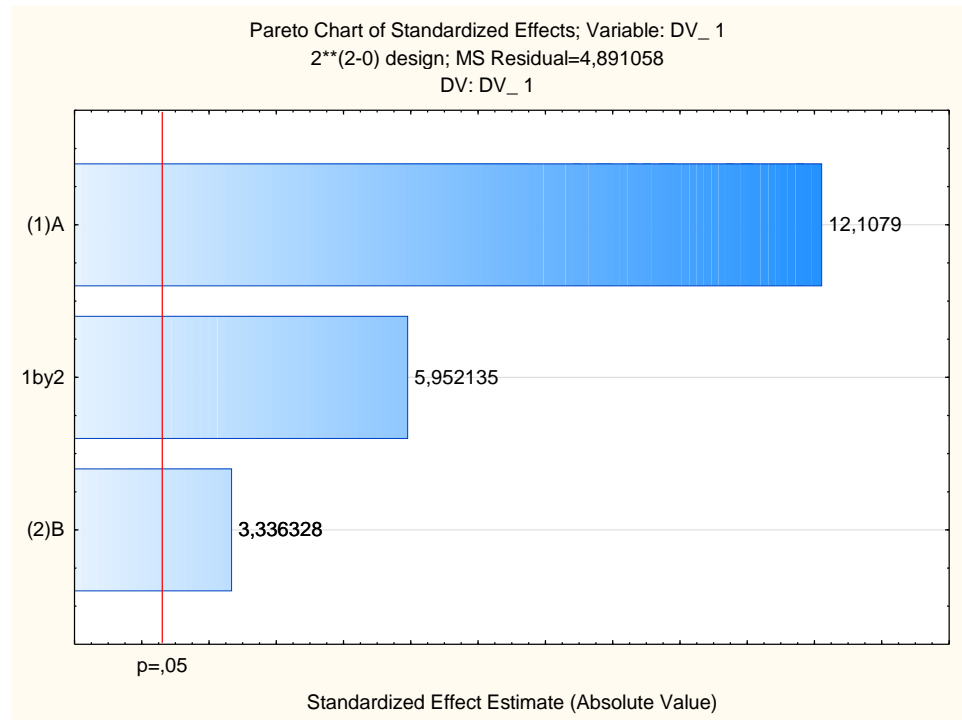

Fonte: Autores.

A partir do Gráfico 2 foi possível concluir que tanto as variáveis capacitor térmico e cobertura como a interação entre elas são significativas, uma vez que, horizontalmente, estes valores estão à frente da linha de rejeição estipulada pelo software de 5\%. Sendo a variável capacitor a mais relevante, seguida da interação com a variável cobertura e por esta. Como as duas variáveis e a interação entre elas são significativas, o modelo linear estatístico do planejamento realizado ficou da forma, 


$$
Y=32,56+7,73 A+2,13 B+3,80 A B+1,28
$$

onde $A$ é a variável capacitor térmico, $B$ é a variável cobertura transparente $A B$ é a interação entre as variáveis $A$ e $B$.

A partir dos gráficos de superfície de resposta e sua projeção no plano ortogonal (Figura 3) do planejamento experimental foi possível determinar a configuração ótima para o coletor solar.

Figura 2: Coletor solar com cobertura de plástico estufa e policarbonato.

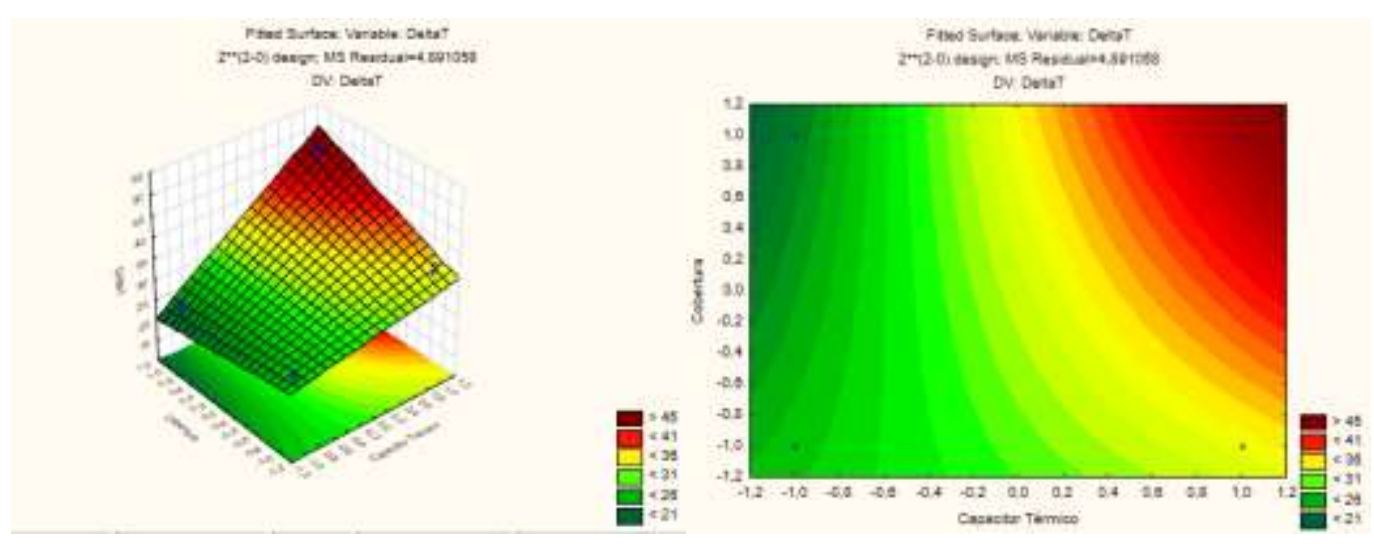

Fonte: Autores.

Como é possível observar na Figura 2, a configuração ótima é obtida quando ambas as variáveis estão no nível superior, ou seja, uso do capacitor térmico e cobertura de policarbonato alveolar.

Em contrapartida, o coletor solar que se apresentou menos eficiente foi o de cobertura de policarbonato e sem capacitor.

Ainda analisando os gráficos de superfície de resposta pode-se observar que o ganho de temperatura na configuração de cobertura transparente codificado por [-1], plástico estufa, apresenta tendência de crescimento quando combinado com o capacitor térmico codificado por [+1], telha de zinco pintada de preto fosco, e que o ganho de temperatura para esta configuração, que é na faixa de $31^{\circ} \mathrm{C}$ a $36^{\circ} \mathrm{C}$, é suficiente para que o processo de secagem solar aconteça quando se faz uso de convecção natural (que segundo Moran et al (2018) a velocidade do ar para um coletor solar sem um sistema de exaustão auxiliar é de $0,2 \mathrm{~m} / \mathrm{s}$ ).

Além disso, optando-se por não usar o capacitor térmico, visto que isso poderia encarecer o equipamento, a melhor opção de cobertura é o plástico estufa, o que tornaria os custos ainda menores.

Na Tabela 3 é apresentado a tabela de ANOVA - Análise de variância do planejamento experimental realizado. 
Tabela 3: ANOVA para o coletor solar.

\begin{tabular}{c|c|c|c|c}
\hline Fonte de Variação & $\begin{array}{c}\text { Soma dos quadrados } \\
\text { (S.Q) }\end{array}$ & $\begin{array}{c}\text { Graus de } \\
\text { liberdade (G.L) }\end{array}$ & $\begin{array}{c}\text { Quadrado da } \\
\text { Média (Q.M) }\end{array}$ & Teste F \\
\hline $\begin{array}{c}\text { Efeito da variável capacitor } \\
\text { térmico }\end{array}$ & 720,44 & 1 & 720,44 & 149,16 \\
\hline $\begin{array}{c}\text { Efeito da variável cobertura } \\
\text { transparente }\end{array}$ & 53,59 & 1 & 53,59 & 11,09 \\
\hline $\begin{array}{c}\text { Efeito da Interação (AxB) } \\
\text { Regressão }\end{array}$ & 174,80 & 1 & 174,80 & 36,19 \\
\hline Resíduos & 948,83 & 3 & 316,28 & $\mathbf{6 5 , 4 8}$ \\
\hline Total & 38,63 & 8 & 4,83 & 1 \\
\hline \multicolumn{7}{c|}{ Coeficiente de Correlação: $\mathbf{R}^{\mathbf{2}} \mathbf{9 6 , 1 0 \%}$} & - & - \\
\hline
\end{tabular}

Fonte: Autores.

Como pode-se observar na tabela 3, o F calculado para a regressão foi de 65,48 e, para este caso o F tabelado é 4,07, então o modelo é significativo e preditivo, ou seja, além de representar adequadamente os experimentos realizados, o modelo pode ser utilizado para fins preditivos afim de prever respostas aproximadas para outras réplicas dos testes realizados.

$\mathrm{Na}$ Tabela 4 estão apresentados os dados referentes às propriedades do ar para os coletores solares sem sistema de exaustão auxiliar.

Tabela 4: Parâmetros do ar para os experimentos.

\begin{tabular}{c|c|c}
\hline Parâmetro & Valor & Unidade \\
\hline Velocidade & 0,2000 & $\mathrm{~m} / \mathrm{s}$ \\
\hline Massa Específica & 1,2754 & $\mathrm{~kg} / \mathrm{m}^{3}$ \\
\hline Calor Específico & 1,0048 & $\mathrm{~kJ} / \mathrm{kg} . \mathrm{K}$ \\
\hline
\end{tabular}

Fonte: Adaptado de Moran et al. (2018).

Na Tabela 5 estão explicitadas as médias de alguns parâmetros como radiação solar média, variação de temperatura, potência útil e o rendimento térmico para cada experimento. 
Research, Society and Development, v. 10, n. 7, e39010716755. 2021

(CC BY 4.0) | ISSN 2525-3409 | DOI: http://dx.doi.org/10.33448/rsd-v10i7.16755

Tabela 5: Parâmetros, potência útil e rendimento térmico de cada experimento.

\begin{tabular}{|c|c|c|c|c|c|}
\hline Experimento & $\begin{array}{c}\text { Datas do } \\
\text { Experimento }\end{array}$ & $\begin{array}{l}\overline{R a d} \\
{\left[\mathrm{~W} / \mathbf{m}^{2}\right]}\end{array}$ & $\begin{array}{l}\overline{\Delta T} \\
{[\mathrm{~K}]}\end{array}$ & $\begin{array}{c}\boldsymbol{P}_{u}=\boldsymbol{m} * c_{p} * \Delta T \\
{[\mathrm{~W}]}\end{array}$ & $\begin{array}{c}\eta_{t}=\frac{P_{u}}{A * I} \\
{[\%]}\end{array}$ \\
\hline \multirow{3}{*}{ EXP1 e Réplicas } & $01 / 11 / 2020$ & 784,50 & 27,83 & 249,38 & 31,79 \\
\hline & $10 / 11 / 2020$ & 838,78 & 24,87 & 223,37 & 26,63 \\
\hline & $30 / 11 / 2020$ & 997,58 & 26,81 & 240,41 & 24,10 \\
\hline \multirow{3}{*}{ EXP2 e Réplicas } & $02 / 11 / 2020$ & 949,07 & 37,20 & 334,61 & 35,26 \\
\hline & $15 / 11 / 2020$ & 1003,37 & 35,35 & 317,56 & 31,65 \\
\hline & $18 / 11 / 2020$ & 953,58 & 30,54 & 271,81 & 28,50 \\
\hline \multirow{3}{*}{ EXP3 e Réplicas } & $10 / 11 / 2020$ & 838,78 & 22,01 & 197,35 & 23,53 \\
\hline & $14 / 11 / 2020$ & 978,26 & 23,00 & 206,33 & 21,09 \\
\hline & $16 / 11 / 2020$ & 978,02 & 24,48 & 218,88 & 22,38 \\
\hline \multirow{3}{*}{ EXP4 e Réplicas } & $18 / 11 / 2020$ & 953,58 & 46,65 & 418,03 & 43,84 \\
\hline & $23 / 11 / 2020$ & 1003,73 & 47,96 & 429,69 & 42,81 \\
\hline & $26 / 11 / 2020$ & 1005,33 & 44,06 & 395,61 & 39,35 \\
\hline
\end{tabular}

Fonte: Autores.

É possível observar que os dados de radiação solar média variam de $784,50 \mathrm{~W} / \mathrm{m}^{2}$ a $1005,33 \mathrm{~W} / \mathrm{m}^{2}$ possuindo média geral de $940,38 \mathrm{~W} / \mathrm{m}^{2}$, desvio padrão de 76,09 e coeficiente de variação de $8,09 \%$. Pode-se observar ainda que os experimentos foram realizados de forma randômica o que torna a análise ainda mais precisa e que o rendimento térmico obtido em cada experimento varia de $21,09 \%$ a $43,84 \%$.

\section{Conclusão}

Com base nos experimentos e nas análises feitas a partir da técnica do planejamento experimental fatorial pode-se inferir que:

- O nível de acurácia dos experimentos, $\mathrm{R}^{2}=96,10 \%$, é considerado satisfatório;

- O modelo linear obtido é significativo e preditivo;

- As duas variáveis estudadas são significativas, bem como a interação entre elas;

- A configuração ótima obtida para o coletor solar foi a com níveis codificados por [+1] para ambas as variáveis analisadas, ou seja, aquele que usa a telha de zinco pintada de preto fosco como capacitor térmico e cuja cobertura transparente é o policarbonato alveolar.

- O coletor solar foi construído com materiais de baixo custo, facilmente encontrados no comércio local, como forma de garantir um equipamento que possa ter sua tecnologia disseminada e que atenda à demanda dos produtores da região;

- Sua operação e manutenção foram executadas sem necessidade de ações de elevada complexidade, o que comprovam sua facilidade de construção e operação, tornando-o uma tecnologia apropriada para inserção no ambiente comercial;

- O rendimento térmico médio para as configurações 1, 2, 3 e 4 do coletor solar são, respectivamente: 27,51, $31,80,22,33$ e 42,00 .

- O maior rendimento do coletor solar foi obtido na configuração otimizada determinada pelo planejamento experimental fatorial realizado. 
- Ficam como sugestões para trabalhos futuros:

- Repetir estes experimentos aplicando a conversão forçada nos coletores;

- Anexar ao coletor solar otimizado uma câmara de secagem e testar experimentalmente na secagem de frutas;

\section{Referências}

Almeida, D. S. F., Chaves, V. F. \& Sant'anna, L. S. (2018). Agricultor Familiar e Produtor Rural no Brasil: os efeitos jurídicos de sua formalização como empresários individuais. Revista Jurídica da Presidência. 20(120).

Brasil, J. L., Vaghetti, J. C. P., Santos Jr, B. R. A., Simon, N. M., Pavan, F. A., Dias, S. L. P. \& Lima, E. C. (2007). Planejamento estatístico de experimentos como uma ferramenta para otimização das condições de biossorção de $\mathrm{Cu}(\mathrm{II})$ em batelada utilizando-se casca de nozes pecã como biossorvente. Química Nova. 30(2), 548-553.

Coelho, B. E. S., Nascimento, M. M., Carvalho, I. R. B., Sousa, K. S. M., Machado, N. S. \& Neto, A. F. (2019). Curva de secagem solar e avaliação físicoquímica da manga "Espada". Journal of Environmental Analysis and Progress. 04(03), 187-194.

Centro de Referência em Energia Solar e Eólica Salvo Brito (2019). http://www.cresesb.cepel.br/.

Cunico, M. W. M., Cunico, M. M., Miguel, O. G. Zawadzki, S. F., Peralta-Zamora, P. \& Volpato, N. (2008). Planejamento fatorial: Uma ferramente estatística valiosa para a definição de parâmetros experimentais empregados na pesquisa científica. Visão acadêmica. 09(01), 23-32.

Fernandes, J. F. S. (2021). Desenvolvimento experimental de um secador solar especial com sílica gel como dessecante do ar de secagem. Dissertação de mestrado. Programa de Pós-Graduação em Engenharia Mecânica, Universidade federal de Campina Grande, Campina Grande.

Freund, J. E. (2006). Estatística aplicada: Economia, administração e contabilidade. (11a ed.), Bookman.

Gomes, Í. A. (2021). Dimensionamento e Otimização de um Secador Solar de Frutas Utilizando o Método do Planejamento Experimental Fatorial. Tese de doutorado. Programa de Pós-Graduação em Engenharia de Processos, Universidade Federal de Campina Grande, Campina Grande.

Grilo, M. B. (2007). Fundamentos da Energia Solar: conceitos básicos e aplicações. Editora da Universidade Federal de Campina Grande - EDUFCG.

Guerra, M. I. S. \& Varella, F. K. O. M. (2014). Análise do desempenho térmico de um sistema de aquecimento solar de baixo custo na cidade de Mossoró/ RN. Revista Holos, 4, 241-268.

Instituto Nacional de Meteorologia. (2020). http:www.inmet.gov.br/portal.

Jacob, P. F. (2016). Estudo do rendimento térmico de um aquecedor solar parabólico redondo para viabilidade de uso comercial e residencial. Dissertação de Mestrado. Faculdade de Engenharia do campus de Guaratinguetá, Universidade Estadual Paulista, Guaratinguetá.

Montgomery, D. C (2017). Design and Analysis of Experiments. (9a ed.), John Wiley \& Sons.

Moran, M. J., Shapiro, H. N., Boettner, D. D. \& Bailey, M. B. (2018). Fundamentals of Engineering Thermodynamics. (8a ed.), John Wiley \& Sons.

Mustayen, A. G. M. B., Mekhilef, S., Saidur, R. (2014). Performance study of different solar dryers: a review. Renewable and Sustainable Energy Reviews, 34, 463-470.

Neves, C. F. C., Schvartzman, M. M. A. M. \& J, E. (2002). Variables search technique applied to gas separation. Química Nova. $25(2)$, $327-329$.

Nunes, A. G. (2016). Desenvolvimento Experimental de um Secador Solar Multienergético para Secagem de Frutas. Tese de Doutorado. Programa de PósGraduação em Engenharia de Processos. Universidade Federal de Campina Grande, Campina Grande.

Oliveira, L. C. T. G., Paes, J. L., Guimarães, C. L. (2018). Avaliação da eficiência do coletor solar plano acoplado em secador híbrido. VII Congresso Brasileiro de Energia Solar. Gramado-RS.

Rodrigues, M. I. \& Iemma, A. F. (2014). Planejamento de Experimentos e Otimização de Processos. (4a ed.), Cárita.

Silva, S. T \& Sousa, N. G. (2020). Uso da energia solar como fonte alternativa para o aquecimento de utilidades: simulação e controle. Research, Society and Development. 9(3). https://doi.org/10.33448/rsd-v9i3.2730

Peralta-Zamora, P., Morais, J. L. \& Nagata, N. (2005). Por que otimização multivariada? Engenharia Sanitária e Ambiental. 10(2), 106-110.

Pereira-Filho, E. R., Poppi, R. J. \& Arruda, M. A. Z. (2002). Employment of factorial design for optimization of pirolisys and atomization temperatures for Al, Cd, Mo and Pb determination by ETAAS. Química Nova. 25(2), 246-253. 